\title{
Coherent atom-trimer conversion in a repulsive Bose-Einstein condensate
}

\author{
H. Jing ${ }^{1}$, J. Cheng ${ }^{2}$, and P. Meystre*1 \\ ${ }^{1}$ Department of Physics, The University of Arizona, Tucson, Arizona 85721, USA \\ ${ }^{2}$ School of Physical Science and Technology, South China University \\ of Technology, Guangzhou 510640, People's Republic of China
}

(Dated: October 11, 2018)

\begin{abstract}
We show that the use of a generalized atom-molecule dark state permits the enhanced coherent creation of triatomic molecules in a repulsive atomic Bose-Einstein condensate, with further enhancement being possible in the case of heteronuclear trimers via the constructive interference between two chemical reaction channels.

PACS numbers: 03.75.Pp, 42.50.-p, 03.70.+k
\end{abstract}

The experimental realization of Bose-Einstein condensates (BEC) in dilute atomic vapors has led to a number of spectacular advances with implications well past the confines of traditional atomic, molecular and optical (AMO) physics [1], and with profound relevance for fields ranging from condensed matter physics to quantum information science. A development of particular relevance for the present study is the generation and probing of ultracold molecular samples and of molecular condensates from atomic BEC, using magnetic Feshbach resonances (FR) and photoassociation (PA) [2-6] to control the dynamics of the system. In particular, it has recently been shown that these two techniques can be combined to achieve the efficient and stable conversion of atoms into molecular dimers [3].

The goal of this paper is to demonstrate theoretically that these techniques can in principle be extended to the generation of molecular trimers. An important new result is that the creation of heteronuclear trimers can be significantly enhanced by the constructive interference of two quantum channels leading to their formation from a two-component atomic condensate. The basic idea is to first create highly excited dimers via a standard FR, and then to couple them to a bound trimer via PA. A key element of the scheme is to exploit a coherent population trapping (CPT) technique to prevent the dimer population from becoming significant throughout the conversion process. Such a scheme has previously been proposed for the creation of molecular dimers [3], and has been theoretically demonstrated to be stable for a broad range of conditions.

This proposed extension of "superchemistry" [6] manipulations from dimers to trimers exploits the existence of three-body bound states in ultracold atomic samples as the scattering length for two-body collisions becomes infinite. As discussed in Ref. [7] this occurs not only for identical particles, a situation first considered by Efimov [8-10], but also for two identical and one different particle. In particular, in the case of two heavy particles and one light particle these authors found that the heavyheavy interaction does not matter, and there is an infinite number of bound trimer states as long as the light-heavy scattering length becomes infinite. In the case of the interaction between one heavy and two light particles, by contrast, there is no infinite number of bound states unless both two-body scattering lengths become simultaneously infinite.

We mentioned that a point of particular interest in the formation of heteronuclear trimers $\mathrm{A}_{2} \mathrm{~B}$ is the role of quantum interferences between the two paths that involve intermediate dimers $\mathrm{A}_{2}$ (path $\mathrm{AA}$ ) and $\mathrm{AB}$ (path $\mathrm{AB}$ ), respectively. To set the stage we consider first the path AA, whereby AA dimers formed via a FR are photoassociated with an atom $\mathrm{B}$ to form the trimer $\mathrm{A}_{2} \mathrm{~B}$ [3]. The essence of the idea is to minimize the occupation of intermediate dimers by exploiting an atom-molecule dark state that permits the direct association of atoms into trimers without the formation of a substantial dimer population in the process [5]. We note that while the proposed scheme is experimentally challenging, recent progress in the manipulation of dimer-atom resonances [11-14] indicates that it might become realizable in the near future. Note also that this process is quite different from a FR-induced dimer-trimer mixture in a resonant condensate [15].

Our model system consists of a Bose-Einstein condensate of atoms coupled to molecular dimers via a FR, these dimers being in turn photoassociated to the atoms to form bound trimers. Denoting the strength of the atomdimer coupling with detuning $\delta$ by $\lambda_{1}^{\prime}$, the Rabi frequency of the PA laser by $\Omega_{1}^{\prime}$ and its detuning by $\Delta$, the dynamics of the system is described at the simplest level by the model Hamiltonian

$$
\begin{aligned}
\hat{\mathcal{H}}= & -\hbar \int d r\left\{\sum_{i, j} \chi_{i j} \hat{\psi}_{i}^{\dagger}(r) \hat{\psi}_{j}^{\dagger}(r) \hat{\psi}_{j}(r) \hat{\psi}_{i}(r)\right. \\
& +\delta \hat{\psi}_{d}^{\dagger}(r) \hat{\psi}_{d}(r)+\lambda_{1}^{\prime}\left[\hat{\psi}_{d}^{\dagger}(r) \hat{\psi}_{a}(r) \hat{\psi}_{a}(r)+h . c .\right] \\
& \left.+(\Delta+\delta) \hat{\psi}_{g}^{\dagger}(r) \hat{\psi}_{g}(r)-\Omega_{1}^{\prime}\left[\hat{\psi}_{g}^{\dagger}(r) \hat{\psi}_{d} \hat{\psi}_{b}+h . c .\right]\right\} .
\end{aligned}
$$

Here, the annihilation operators $\hat{\psi}_{i}(r)$, where the indices $i, j=a, b, d, g$ stand for atoms (A and B), dimers and trimers, satisfy bosonic commutation relations, and the 
collision terms proportional to $\chi_{i j}$ describe $s$-wave collisions between these species. (Trimer formation via the path $\mathrm{AB}$ only can be investigated in a similar fashion.) The nonlinear bound-bound coupling between dimers and trimers is typically induced by a narrow-frequency, continuous-wave PA laser, for which the Franck-Condon factor can be calculated by resonant scattering theory $[2$, $11]$ and may be tuned by pumping methods [16-17].

We assume in the following that the main features of the dynamics are adequately described by a mean-field analysis, $\hat{\psi}_{i} \rightarrow \sqrt{n} \psi_{i}$, where $n$ is the initial atomic density. In this limit, the system is described by the equations of motion $(\hbar=1)$

$$
\begin{aligned}
\frac{d \psi_{a}}{d t}= & 2 i n \sum_{j} \chi_{a j}\left|\psi_{j}\right|^{2} \psi_{a}+2 i \lambda_{1} \psi_{d} \psi_{a}^{*}, \\
\frac{d \psi_{b}}{d t}= & 2 i n \sum_{j} \chi_{b j}\left|\psi_{j}\right|^{2} \psi_{b}-i \Omega_{1} \psi_{d}^{*} \psi_{g}, \\
\frac{d \psi_{d}}{d t}= & -(\gamma-i \delta) \psi_{d}+2 i n \sum_{j} \chi_{d j}\left|\psi_{j}\right|^{2} \chi_{d} \psi_{d}+i \lambda_{1} \psi_{a}^{2} \\
& -i \Omega_{1} \psi_{b}^{*} \psi_{g}, \\
\frac{d \psi_{g}}{d t}= & 2 i n \sum_{j} \chi_{g j}\left|\psi_{j}\right|^{2} \psi_{g}+i(\Delta+\delta) \psi_{g}-i \Omega_{1} \psi_{d} \psi_{b},
\end{aligned}
$$

where $\lambda_{1}=\lambda_{1}^{\prime} \sqrt{n}, \Omega_{1}=\Omega_{1}^{\prime} \sqrt{n}$ and the decay rate $\gamma$ accounts for the loss of untrapped dimers. To reduce these losses we exploit a CPT technique that relies on the existence of an approximate atom-molecule dark state. Such techniques are well known in the case of linear systems, where they permit the transfer of population from an initial to a final state via an intermediate state that remains unpopulated at all times. This is the basis for stimulated Raman adiabatic passage (STIRAP), which achieves this goal via a so-called counter-intuitive sequence of pulses [2].

CPT and STIRAP rely explicitly on the validity of the adiabatic theorem, which applies only to linear systems. Unfortunately, in the situation at hand two-body collisions render the system nonlinear, and it is not immediately obvious that STIRAP still works in that case. This problem has recently been investigated in Ref.[4], which shows that an approximate adiabatic condition can still be achieved by linearizing the nonlinear system around the intended adiabatic evolution. If the eigenfrequencies of the linearized system are real, the associated "normal modes" will not grow in time, and the system is stable. Hence a system initially prepared in a CPT state will approximately remain in that state at all times, although the adiabaticity condition may be difficult to fulfill at the later stages of the evolution. In contrast, if the eigenvalues of the linearized problem are complex, the system is dynamically unstable for some parameter values [3] under which adiabaticity breaks down.

For the specific case of trimer formation it is easily shown that under the generalized two-photon resonance condition

$$
\begin{aligned}
\Delta= & -\delta+2\left(2 \chi_{a g}+\chi_{b g}-\chi_{g g}\right) n N_{g, s} \\
& +\left(4 \chi_{a a}-2 \chi_{a g}+4 \chi_{a b}+\chi_{b b}-\chi_{b g}\right) n N_{a, s}
\end{aligned}
$$

eqs. (11) admit a steady-state solution with no dimer population,

$$
N_{g, s}=\frac{1}{3}\left(\frac{k\left(\lambda_{i} / \Omega_{i}\right)^{2}}{1+k\left(\lambda_{i} / \Omega_{i}\right)^{2}}\right),
$$

where $i=1$ and $k=4$. (For the path AB, Eq. (3) remains the same, but with $i=2$ and $k=1$, so that $N_{g, s}^{\mathrm{AB}}<N_{g, s}^{\mathrm{AA}}$ for the same external parameters. Note that $N_{d, s}=0$ and the same two-photon resonance condition holds in both cases.)

This suggests that approximate CPT dynamics such that the dimer population $N_{d}$ remains small at all times can be achieved for an appropriate "counter-intuitive" time dependence of the laser detuning $\Delta[3]$ (see also Ref. [18]). Additionally, it may be possible to exploit a feedback technique [19] to further stabilize this process.
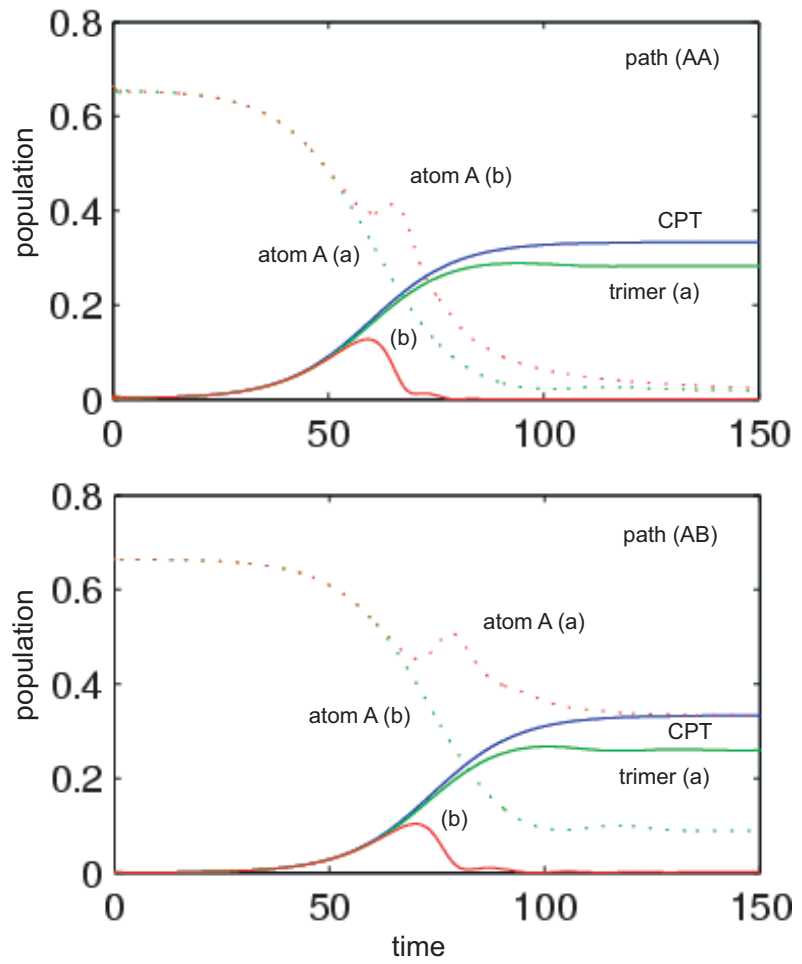

FIG. 1: (Color online) Heteronuclear trimer population for (a) $\delta=-3$ (in green) or (b) $\delta=3$ (in red) and their corresponding numbers of atom A. Time is in units of $\lambda_{l}^{-1}$, and $\gamma=1$. The dimer population $\left(\mathrm{A}_{2}\right.$ or $\left.\mathrm{AB}\right)$ remains essentially zero at all times and is not shown. The line labelled "CPT" shows the ideal, analytically derived trimer population. The evolution of the atoms B is also not shown.

Figure 1 shows a numerical simulation of the formation of the heteronuclear trimers $\mathrm{A}_{2} \mathrm{~B}$, when considering the 
channels AA and AB separately. In this specific example the atom $\mathrm{A}$ is ${ }^{23} \mathrm{Na}$, with the $s$-wave scattering length $3.4 \mathrm{~nm}$, and atom B is ${ }^{87} \mathrm{Rb}$, with an $s$-wave scattering length of $5.77 \mathrm{~nm}, \lambda_{l}=4.718 \times 10^{4} \mathrm{~s}^{-1}(l=1,2)$, and

$$
\Omega_{l}(t)=\Omega_{l, 0} \operatorname{sech}(t / \tau),
$$

with $\Omega_{l, 0} / \lambda_{l}=20, \lambda_{l} \tau=20$. The $s$-wave scattering length for Na-Rb collisions, which depends on the details of the interatomic potential, is not well known. Here we take $\chi_{a}=0.3125, \chi_{b}=0.5303, \chi_{a b}=0.4214$ and the other collisions parameters are taken as 0.0938 (in units of $\lambda_{l} / n$ ) [20]. Very little is known about the scattering lengths of collisions involving molecular trimers, so we have carried out simulations with several sets of plausible parameters $[20]$. We found that the stable formation of trimers is always possible for a range of values of the detuning $\delta$.

The curves labelled (a) in Fig. 1 give one such example for $\delta=-3$. Their general features resemble those of Fig. 2 in Ref. [4], which corresponds however to the creation of dimers rather than trimers. In particular, we observe a similarly increasing departure of the population transfer from the CPT solution as time evolves. Just as is the case for dimer formation [3], the association of atoms into trimers is characterized by the existence of regions with unstable dynamics. This is for instance the case for for $\delta=3$ (curves (b) in Fig. 1.) As expected, the two reaction channels lead to different dynamical behaviors. In particular, the $\mathrm{AB}$ channel leaves a significantly larger number of atoms $\mathrm{A}$ in the sample at the end of the conversion process, and hence results in a lower yield of heteronuclear trimers.

We conclude the discussion of the single-channel cases by noting that we have also studied the full quantum dynamics of heteronuclear or homonuclear trimer formation in the short-time limit using a $c$-number positive- $P$ representation approach [6]. We find that in that limit the quantum dynamics reproduces the CPT dimer production predicted by the mean-field theory [21], and quantum noise-induced trimer damping occurs only near a total atom-trimer conversion $[6,21]$.

We now proceed to demonstrate that when acting in concert, the two channels can yield a significantly larger conversion rate and approach the ideal CPT yield of $1 / 3$, see Fig. 2. Here, the coexistence of the two channels provides considerable additional flexibility in approaching the ideal CPT value for trimer formation. Note however that this approach either requires an accidental coincidence of FRs for the $\mathrm{A}_{2}$ and $\mathrm{AB}$ dimer formation, or might be realizable in other cases by applying a magnetic field gradient [22] across the coexisting A and B condensates.

The mean-field description of the two-channel situation is a straightforward extension of the single-channel case. Requiring as before that the number of dimers remains equal to zero, the CPT steady-state number of trimers is

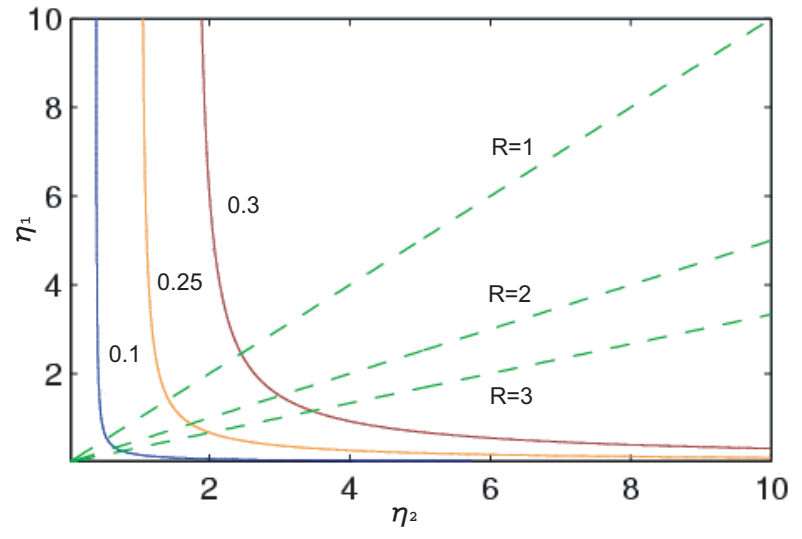

FIG. 2: (Color online) Normalized CPT trimer population as a function of $\eta_{l}=\lambda_{l} / \Omega_{l}, l=1,2$. Also shown are three values of $R=\eta_{2} / \eta_{1}$. At constant ratios $\Omega_{2}(t) / \Omega_{1}(t)$ the counterintuitive evolution of the system is along lines of constant $R$ starting from the origin.

then

$$
N_{g, s}=\frac{\left(\lambda_{1} / \Omega_{1}\right)\left(\lambda_{2} / \Omega_{2}\right)^{2}}{\lambda_{1} / \Omega_{1}+\lambda_{2} / \Omega_{2}+3\left(\lambda_{1} / \Omega_{1}\right)\left(\lambda_{2} / \Omega_{2}\right)^{2}},
$$

where the asymmetry between the two channels results from the fact that the intermediate dimer involves two undistinguishable particles in the first one and two distinguishable particles in the second case.

Figure 2 plots the steady-state trimer number $N_{g, s}$ as a function of $\eta_{l}=\lambda_{l} / \Omega_{l}, l=1,2$, as well as the parameter

$$
R=\eta_{2} / \eta_{1}
$$

(Note that there is no CPT solution for $R<0$.) As the STIRAP PA pulses $\Omega_{1}(t)$ and $\Omega_{2}(t)$ are applied, $\eta_{1}$ and $\eta_{2}$ increase and if the ratio of their amplitudes remains constant the system evolves along a line of constant $R$. To determine whether an optimum choice of $R$ permits to approach the ideal trimer population of $1 / 3$ under the non-ideal STIRAP conditions of our nonlinear system we have solved numerically the mean-field equations of motion of the system for various values of $R \in[1,3]$ (see Fig. 3 ), using the same parameters as in the single-channel case.

We found numerically that $R=2$ leads to a trimer production that most closely approaches the ideal CPT solution, and is significantly larger than in the singlechannel situation of Fig. 1. This is illustrated in Fig.3c for $\delta=0$ and $\delta=-3$. Note the insensitivity of trimer production to the detuning in that case. These results should be contrasted to Fig. 3b, which shows the evolution of the trimer population in case $R=1$, again for $\delta=0$ and $\delta=-3$. Here, the trimer production is very significantly reduced, and depends strongly on the value of the detuning. Similar results have been obtained for the other values of $R$ and the other sets of collisions parameters [20] that we have considered. 

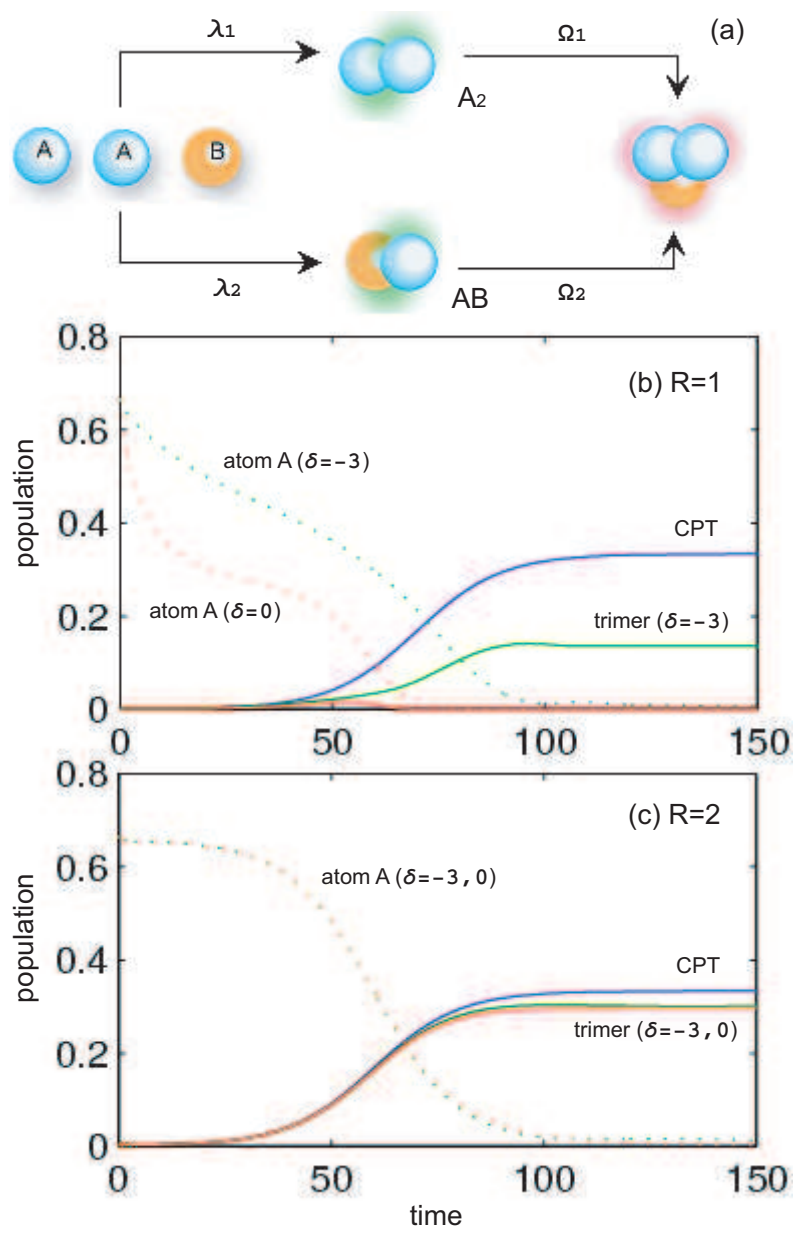

FIG. 3: (Color online) (a) Two-channel generation of heteronuclear trimers. The time dependence of the populations of the trimers (solid) and of atom $A$ (dotted) are shown for $\delta=-3$ and $\delta=0$ and (b) $R=1$ or (c) 2 . The CPT value of trimers is also plotted. $\chi_{d_{1} d_{2}}=0$ and $d_{1,2}$ denotes $\mathrm{A}_{2}$ or $\mathrm{AB}$.

We observe also that in the two-channel case the trimer population can reach a transient value that is larger than its final value. This suggests that maintaining a constant ratio $R$ during the evolution of the system may not be optimal. Future work will use genetic algorithms to determine the optimum time dependence of $R(t)$ for maximum trimer production.

In conclusion, we have shown that a STIRAP scheme based on Feshbach-assisted photoassociation, which has previously been shown to result in the production of ultracold molecular dimers [3], can be extended to the generation of molecular trimers, and that in the case of heteronuclear molecules the interference between two formation channels can lead to a significant enhancement of trimer production. Future work will study further ways to optimize this scheme, and will give a quantummechanical description of the system, in particular with the goal of understanding the role of quantum fluctuations in the early stages of trimer production and the quantum statistics of the trimer field. We also plan to study the stability properties and the adiabatic geometric phase of the system [4], the superchemistry reaction $\mathrm{A}_{2}+\mathrm{B} \rightarrow \mathrm{AB}+\mathrm{B}$ via a dark state technique, and possibly trimer creation or amplification in an optical lattice [23]. While experiments along the lines of this analysis promise to be challenging, recent progress in Efimov superchemistry $[9-12,15]$ and in the manipulation of dimer-atom resonances [11-14] indicates that they may become possible in the not too distant future.

This research is supported in part by the US Office of Naval Research, by the US National Science Foundation, by the US Army Research Office, by the Joint Services Optics Program, by the National Aeronautics and Space Administration and by the NSC and SCUT. The authors thank Dr. M. Bhattacharya for helpful discussions, and Dr. P. Julienne for bringing Ref. [7] to their attention.

* Electronic address: pierre.meystre@optics.arizona.edu

[1] J. R. Anglin and W. Ketterle, Nature (London) 416, 211 (2002); P. Meystre, Atom Optics (Springer-Verlag, 2001).

[2] T. Köhler, K. Góral, and P. S. Julienne, Rev. Mod. Phys. 78, 1311 (2006); K. M. Jones, et al., ibid. 78, 483 (2006).

[3] H.-Y. Ling, H. Pu, and B. Seaman, Phys. Rev. Lett. 93, 250403 (2004).

[4] H. Pu, et al., Phys. Rev. Lett. 98, 050406 (2007).

[5] K. Winkler, et al., Phys. Rev. Lett. 95, 063202 (2005).

[6] J. J. Hope and M. K. Olsen, Phys. Rev. Lett. 86, 3220 (2001).

[7] R. Amado and J. Noble, Phys. Rev. D 5, 1992 (1972).

[8] V. Efimov, Phys. Lett. B 33, 563 (1970); Sov. J. Nucl. Phys. 12, 589 (1971); Com. Nucl. Phys. 19, 271 (1990).

[9] T. Kraemer, et al., Nature (London) 440, 315 (2006).

[10] M. Y. Yamashita, et al., Phys. Rev. A 68, 033406 (2003).

[11] P. Soldán, et al., Phys. Rev. Lett. 89, 153201 (2002); M. T. Cvitaŝ, et al., ibid. 94, 200402 (2005).

[12] E. Nielsen, H. Suno and B. D. Esry, Phys. Rev. A 66, 012705 (2002).

[13] M. Mackie, et al., Eur. Phys. J. D 31, 273 (2004).

[14] C. Ospelkaus, et al., Phys. Rev. Lett. 97, 120402 (2006).

[15] E. Braaten, H.-W. Hammer, and M. Kusunoki, Phys. Rev. Lett. 90, 170402 (2003).

[16] P. D. Lett, et al., Phys. Rev. Lett. 71, 2200 (1993).

[17] F. Colavecchia, et al., J. Chem. Phys. 118, 5484 (2003).

[18] K.-P. Marzlin, W. Zhang, and E. M. Wright, Phys. Rev. Lett. 79, 4728 (1997).

[19] J. Cheng, et al., Phys. Rev. A 73, 035601 (2006).

[20] For example, in the Thomas-Fermi approximation, we can take $\mathrm{Na}-\mathrm{Rb}$ scattering length as half the critical value $\left[\left(M_{\mathrm{Na}} M_{\mathrm{Rb}}\right)^{1 / 2} /\left(M_{\mathrm{Na}}+M_{\mathrm{Rb}}\right)\right]\left(a_{\mathrm{Na}} a_{\mathrm{Rb}}\right)^{1 / 2}$ for the stable coexistence of $\mathrm{Na}$ and Rb BECs. Here $M_{\mathrm{Na}, \mathrm{Rb}}$ and $a_{\mathrm{Na}, \mathrm{Rb}}$ denote the mass and scattering length of $\mathrm{Na}$ and $\mathrm{Rb}$ atoms, respectively. Thus, by using $\chi_{a b}=0.0832$ and assuming the other collisions parameters in both cases to be those of atom A, except for $\chi_{\sigma \varsigma}=-1.4583(\sigma=a, b$ and $\varsigma=d, g$ ), we can reach a similar result as Fig. 1 (with fewer numbers of trimers).

[21] Details of the derivations will be published elsewhere. 
[22] H. Xiong, et al., Phys. Rev. Lett. 95, 120401 (2005).

(2004).

[23] F. Zhou and G. W. Semenoff, Phys. Rev. Lett. 97, 180411

(2006); C. P Search and P. Meystre, ibid. 93, 140405 